\title{
BMJ Open Surveillance Systems from Public Health Institutions and Scientific Societies for Antimicrobial Resistance and Healthcare-Associated Infections in Europe (SUSPIRE): protocol for a systematic review
}

\author{
María Núñez-Núñez, ${ }^{1,2,3}$ María Dolores Navarro, ${ }^{1,2}$ Panagiota Gkolia, ${ }^{4}$ \\ Nithya Babu Rajendran, ${ }^{4}$ María Dolores del Toro, ${ }^{1,2}$ Andreas Voss, ${ }^{5}$ \\ Mike Sharland, ${ }^{6}$ Frangiscos Sifakis, ${ }^{7}$ Evelina Tacconelli, ${ }^{4}$ Jesús Rodríguez-Baño, ${ }^{1,2}$ \\ and Members of the EPI-NET group on behalf of COMBACTE-MAGNET Consortium
}

To cite: Núñez-Núñez M, Navarro MD, Gkolia P, et al. Surveillance Systems from Public Health Institutions and Scientific Societies for Antimicrobial Resistance and Healthcare-Associated Infections in Europe (SUSPIRE): protocol for a systematic review. BMJ Open 2017:7:e014538.

doi:10.1136/bmjopen-2016014538

- Prepublication history and additional material is available. To view please visit the journal (http://dx.doi.org/ 10.1136/bmjopen-2016014538).

Received 10 October 2016 Revised 30 January 2017 Accepted 31 January 2017

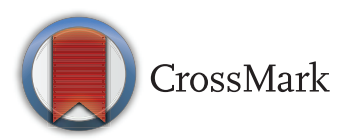

For numbered affiliations see end of article.

Correspondence to María Núñez-Núñez; mnunez.pharm@gmail.com

\section{ABSTRACT}

Introduction: The worldwide spread of antimicrobial resistance is now recognised as a global public health threat. Owing to the geographical heterogeneity, complexity and continuously evolving dynamics of resistant organisms and genes, surveillance is a key tool for understanding, measuring and informing actions in the fight against this problem. To date there is no harmonisation of key indicators or of methodologies used to obtain them.

Methods and analysis: The main objective of this project is to systematically review and analyse the current publicly available surveillance activities on antimicrobial resistance and healthcare-associated infections in Europe. Eligible activities are those endorsed by regional, national or transnational health organisations and scientific societies providing data on a periodic basis. Grey and peer-reviewed literature will be searched with no language restrictions. Three independent reviewers will perform a two-step selection process using a previously piloted, tailored electronic data extraction form. Descriptive summaries and tables of all relevant findings will be performed and reported according to PRISMA guidelines.

Ethics and dissemination: We did not seek ethical approval for this study because the data to be collected are not linked to individuals. Data will be presented at international conferences and published in peerreviewed journals.

Trial registration number: CRD42016033867.

\section{INTRODUCTION}

The worldwide spread of antimicrobial resistance (AMR) is now recognised as a global public health threat. ${ }^{12}$ Owing to the geographical heterogeneity, complexity and continuously

\section{Strengths and limitations of this study}

- Wide scope search strategy including peerreviewed and grey literature.

- No language restriction and study protocol designed by a multidisciplinary team within a multinational collaborative consortium.

- Surveillance systems for which the methodology is not publicly available will be missed.

- The review will rely on the data provided in the accessible sources, while some surveillance activities might have changed after their publication.

- Despite following a carefully prepared search strategy, some surveillance systems or specific information from them may not be found. However, this will indicate a problem in the accessibility to the information.

evolving dynamics of resistant organisms and genes, surveillance is a key tool for understanding, measuring and informing actions in the fight against this problem. Indeed, surveillance systems for AMR have been developed by most national health systems and transnational organisations. $^{3-5}$ Adequate characterisation of the burden of disease caused by resistant pathogens, their impact on patient outcomes and the areas and patient populations with the highest incidences are critical for identifying medical needs, establishing treatment protocols and efficiency of design for randomised controlled trials. The situation with healthcare-associated infections (HAI), which are often closely associated with AMR, is similar. 
An important problem for the surveillance of AMR and HAI is heterogeneity of the scope, focus, objectives, methodology, resources and reporting across the different regions and countries, despite the efforts of institutions such as the European Centre for Disease Prevention and Control (ECDC), Centers for Disease Control and Prevention (CDC) or the WHO. ${ }^{3-5}$ The ECDC conducted a comprehensive review of the current situation and implications of HAIs across Europe; these authors highlighted that while some progress has been achieved in recent years, there are still substantial intercountry and intracountry differences in surveillance methods and concluded that greater emphasis be placed on harmonisation. ${ }^{5}$

The objectives of SUSPIRE are to systematically review and analyse the surveillance activities endorsed by national or transnational health organisations and scientific societies that are performed in the European Union and European Economic Area (EU/EAA) and to provide regular data on HAI and AMR. The final objective of this effort is to provide recommendations that can be used for the harmonisation of surveillance systems.

\section{METHODS}

\section{Eligibility criteria}

SUSPIRE targets surveillance systems endorsed by regional, national or transnational health organisations or scientific societies in EU/EEA countries that are in place for the purpose of providing regular data on AMR and/or HAI.

\section{Information sources and search strategy}

A comprehensive search strategy for searching grey and peer-reviewed literature will be performed until 30 November 2016, using the Google search engine for the first task, and combining the following terms in the local languages of the various European countries or regions (depending on whether the public health providers are centralised/national or decentralised): 'Antimicrobial resistance' AND/OR 'Hospital-associated' OR 'Hospital-acquired' OR 'Nosocomial' AND 'Surveillance' AND 'epidemiology' OR 'prevalence' OR 'incidence'.

Sources of peer-reviewed literature to be searched include: PubMed, Embase, Scopus (Elsevier Science) and Web of Science (Thomson, ISI), using the same subject headings (MeSH). A draft PubMed search strategy is included in online supplementary appendix 1.

Articles available until 30 June 2016 will be included. 'Zoonotic' OR 'food-borne' OR 'outbreak' will be excluded in all cases, and 'human data' will be imposed on the search whenever possible.

We will also review the official health-related websites of national and transnational health organisations and scientific societies, such as the ECDC, WHO Europe, regional and national European governments, and of relevant scientific societies, such as ESCMID (European Society of Clinical Microbiology and Infectious Diseases;
ISID (International Society for Infectious Diseases); IEA (International Epidemiological Association); ESICM (European Society of Intensive Care Medicine) or ERS (European Respiratory Society), among others. Additional data will be obtained from references in retrieved articles and data provided by stakeholders following the request to complete the catalogue of surveillance systems and reports on AMR and HAI.

\section{Inclusion and exclusion criteria}

The documents retrieved will be assessed for their content and the inclusion and exclusion criteria shown in box 1 applied for those documents to be finally included in the review.

No language restrictions will apply; local experts, identified by the European Committee on Infection Control (EUCIC) of the European Society of Clinical Microbiology and Infectious Diseases, will be consulted.

\section{Data management, selection process and data extraction}

The literature search results will be uploaded to EndNote X7 software. Two independent reviewers will perform a two-step selection process. Titles and abstracts (if available) of the retrieved documents will be initially assessed and non-relevant documents excluded. The full text of potentially eligible documents will then be obtained and assessed for relevance or duplication against predefined selection criteria.

Data will be extracted independently by two reviewers, using a tailored electronic data extraction form to be piloted beforehand on a representative sample. The

\section{Box 1 Eligibility criteria for surveillance systems}

\section{Inclusion criteria}

- European surveillance systems, or including European data

- Epidemiological surveillance studies collecting data for at least five consecutive years from three or more sites, and with at least one data collection year within the last 10 years

- Promoted or endorsed by national or transnational health organisations and scientific societies

- Providing, or with the intention to provide, data on a periodic basis

- Providing data on at least one of the following: objective, scope (eg, hospital and healthcare-associated infections/pathogens), design and methodological issues

- Published scientific and 'grey' literature

- Human data

- No age/language restriction

Exclusion criteria

- Animal, environmental or food data only

- Epidemiological studies or reports whose main objective is different from that of providing surveillance data

- Epidemiological studies or surveillance data promoted by private companies

- Outbreak reports

- Systems that are 'inactive' (not providing any information) for the last 10 years 
Table 1 Summary list of variables collected

\begin{tabular}{|c|c|}
\hline Core element & Variable \\
\hline \multirow[t]{8}{*}{ Program } & System name \& acronym* \\
\hline & Title \& author/s name \\
\hline & Location and magnitude \\
\hline & Status: Active/inactive ${ }^{*} \&$ completed/ongoing† \\
\hline & Coordinating organization and resources \\
\hline & Focus: Type of activity (HAI, AMR) \\
\hline & Link: System website/journal article \\
\hline & Update year \\
\hline \multirow[t]{4}{*}{ Population } & Demographics of population covered (age, gender) \\
\hline & Comorbidities and risk factors \\
\hline & Inclusion/exclusion criteria† \\
\hline & Type and details of healthcare facilities \\
\hline \multirow[t]{5}{*}{ Microbiology } & Specimen type and carriers tested \\
\hline & Duplicates policy \\
\hline & Clinical value of the sample \\
\hline & Pathogens, sources and acquisition \\
\hline & $\begin{array}{l}\text { Microbiological methods for the identification and characterization of mechanisms of } \\
\text { resistanceAntibiotics tested and resistance mechanisms }\end{array}$ \\
\hline $\begin{array}{l}\text { Methodology and indicators } \\
\text { used }\end{array}$ & $\begin{array}{l}\text { Clinical criteria and microbiological definitions used, Structure, process and outcome } \\
\text { indicators reported, Measurement frequency of relevant indicators, Reporting of source } \\
\text { data (volunteer/compulsory) }\end{array}$ \\
\hline $\begin{array}{l}\text { Data analysis, reporting and } \\
\text { dissemination }\end{array}$ & $\begin{array}{l}\text { Source of data and data collection systems, Quality assessments }{ }^{*} \text { Stratification of reported } \\
\text { data, Reporting type and frequency, Dissemination of data* }\end{array}$ \\
\hline
\end{tabular}

same core data will be extracted from systems and studies including specific details about the following elements: programme, population, microbiology, methodology, quality indicators and data. A detailed list of variables is presented in table 1 .

Disagreements between reviewers will be resolved by consultation with a third reviewer.

\section{Quality assessment}

There are several generic guidelines for evaluating human public health surveillance, and these typically include assessing a series of attributes, such as flexibility, acceptability and timeliness, using a combination of quantitative and qualitative techniques. The quality of the surveillance systems included in our review will be assessed on the basis of the attributes recommended in the Centres for Disease Prevention and Control guidelines for evaluating public health surveillance systems. ${ }^{6}$

The protocol was developed following the recently released PRISMA-P guidelines, and the review will be reported in accordance with the PRISMA statement. ${ }^{7}$

\section{Data synthesis and descriptive analysis}

The data synthesis phase will involve collating and summarising the results in the form of a table that indicates the core characteristics of the systems and studies: type of activity, isolate source, population, phenotypes and mechanisms of resistance, definitions of acquisition, indicators and quality assessments. Frequency distributions expressed as percentages (\%) will be calculated for each variable and displayed graphically. Analysis will be stratified by country, surveillance type (system/study) and activity (HAI/AMR).

\section{Dissemination}

Data will be presented at international conferences and published in peer-reviewed journals.

\section{DISCUSSION}

Following the original SENIC studies in the 1970s, surveillance has been recognised as a key component of quality assurance in general and of infection control in particular. ${ }^{8}$ Since then, surveillance procedures and systems have evolved in accordance with increased awareness of the complexity and importance of HAIs as a patient safety concern ${ }^{9}$ and the rise of AMR. Most countries started surveillance systems for HAIs during the 1980s and 1990s. A series of articles published in 2001 showed how heterogeneous the surveillance activities performed in European countries at that time were. ${ }^{10}$ Over recent decades, the ECDC has been extraordinarily active in working towards the homogenisation of definitions, procedures and systems developed. ${ }^{36}$ In 2008, the ECDC conducted a review highlighting that there were still significant limitations to surveillance systems across European countries. ${ }^{11}$ The aim of SUSPIRE is to provide an update of the situation, with the additional purpose of specifically evaluating particular aspects of the 
definitions, methods and quality assessment, as well as the infections and microorganisms/resistances.

AMR surveillance has traditionally focused on either the percentage of particular pathogens that are resistant to certain antimicrobial agents and/or rates of isolation of relevant resistant bacteria. However, some potentially important aspects, such as the specific populations or patients in which these infections occur, types of infection and their outcomes have not usually been considered because of the additional complexity and resources needed. Nevertheless, these are key aspects for informing management decisions about the prioritisation and provision of resources required to address the problem and determining the most urgent areas for research. Furthermore, the way that national surveillance systems are conceptually set up at present may be too slow to provide useful information for immediate global action in situations of the emergence of new resistant pathogens or the further spread of previously known ones, as is shown by the recent spread of carbapenemaseproducing Enterobacteriaceae in many European countries and across borders. ${ }^{12}$ Therefore, specific evaluation of AMR surveillance systems and the gaps that exist is required in order to detect areas for improvement.

The objectives of the COMBACTE-MAGNET consortium, funded by the European Union and, in kind, by the European Federation of Pharmaceutical Industries Association (EFPIA) through the Innovative Medicines Initiative, include building a European network by engaging representatives from the major EU financed projects, stakeholder experts and industry in order to develop a consensus programme and a homogeneous approach to current and future epidemiological surveillance strategies in Europe. The SUSPIRE project will work to achieve this objective.

\section{Author affiliations \\ ${ }^{1}$ Unit of Infectious Disease, Microbiology, and Preventive Medicine, Institute of Biomedicine of Seville (IBIS)/University Hospital Virgen Macarena-Virgen del Rocío/Spanish National Research Council (CSIC), Seville, Spain \\ ${ }^{2}$ Departamento de Medicina, Universidad de Sevilla, Seville, Spain \\ ${ }^{3}$ Department of Pharmacy, University Hospital Virgen Macarena, Seville, Spain \\ ${ }^{4}$ Infectious Diseases, Internal Medicine 1, DZIF Center, Tuebingen University Hospital, Tuebingen, Germany \\ ${ }^{5}$ Department of Medical Microbiology, Radboud University Medical Centre, Nijmegen, The Netherlands \\ ${ }^{6}$ Paediatric Infectious Diseases Research Group, St George's University \\ London, London, UK \\ ${ }^{7}$ AstraZeneca LP, Gaithersburg, Maryland, USA}

Acknowledgements The research leading to these results was conducted as part of the COMBACTE-MAGNET consortium. For EPINET members and further information, please refer to http://www.COMBACTE.com. The authors thank Virginia Palomo for her administrative assistance in the development of the protocol.

Contributors JR-B conceived the study, led the development of the protocol, provided supervision and mentorship to MN-N who wrote the first draft, coordinated and integrated comments from coauthors and together with $J R-B$ is the guarantor of the manuscript. MDN contributed with the selection of variables, and NBR contributed with the development of search strategy. MS and MDdT provided specific expertise on paediatric and surgical site infections variables, respectively. AV contributed with the selection of variables, and FS contributed with development of the protocol and variables selection. ET and PG also provided specific expertise on epidemiology and contributed to the development of search strategy. All authors critically reviewed successive drafts of the manuscript, provided important intellectual input and approved the final version for publication.

Funding This research project receives support from the Innovative Medicines Initiative Joint Undertaking under grant agreement no. 115737 resources of which are composed of financial contribution from the European Union Seventh Framework Programme (FP7/2007/2013) and EFPIA companies in kind contribution. JRB also received funding for research from Ministerio de Economía y Competitividad, Instituto de Salud Carlos III—cofinanced by European Development Regional Fund 'A way to achieve Europe' ERDF, Spanish Network for the Research in Infectious Diseases (REIPI RD12/0015).

Competing interests FS is an employee of AstraZeneca/Medimmune, an EFPIA (European Federation of Pharmaceutical Industries and Association) member company in the IMI JU. Costs related to research contributions by FS are borne by AstraZeneca/Medimmune and considered in-kind contribution under the IMI JU scheme. Rest of authors has declared no conflict of interest related to this paper.

Provenance and peer review Not commissioned; externally peer reviewed.

Open Access This is an Open Access article distributed in accordance with the Creative Commons Attribution Non Commercial (CC BY-NC 4.0) license, which permits others to distribute, remix, adapt, build upon this work noncommercially, and license their derivative works on different terms, provided the original work is properly cited and the use is non-commercial. See: http:// creativecommons.org/licenses/by-nc/4.0/

\section{REFERENCES}

1. European commission. Action plan against the rising threats from Antimicrobial Resistance. Communication from the commission to the European parliament and the council. Brussels, September 2011. http://ec.europa.eu/dgs/health_consumer/docs/ communication_amr_2011_748_es.pdf

2. ECDC/EMEA Joint technical report. The bacterial challenge: time to react. Ref. EMEA/576176/2009. Stockholm, September 2009. ISBN 978-92-9193-193-4.

3. European Centre for Disease Prevention and Control (ECDC), website available on. http://ecdc.europa.eu/en/activities/surveillance/ Pages/index.aspx (date last accessed September 2016).

4. Centers for Disease Control and Prevention (CDC). http://www.cdc. $\mathrm{gov} / \mathrm{nh}$ sn (date last accessed September 2016).

5. World Health Organization (WHO). http://www.who.int/topics/public health_surveillance/en/ (date last accessed September 2016).

6. European Centre for Disease Prevention and Control (ECDC). Data quality monitoring and surveillance system evaluation-A handbook of methods and applications. Stockholm, September 2014. http:// ecdc.europa.eu/en/publications/publications/data-qualitymonitoringsurveillance-system-evaluation-sept-2014.pdf

7. Shamseer L, Moher D, Clarke M, et al., PRISMA-P Group. Preferred reporting items for systematic review and meta-analysis protocols (PRISMA-P) 2015: elaboration and explanation. BMJ 2015;349: g7647.

8. Haley RW, Culver DH, White JW. The efficacy of infection surveillance and control programs in preventing nosocomia infections in US hospitals. Am J Epidemiol 1985;121:182-205.

9. Burke JP. Infection control-a problem for patient safety. N Engl J Med 2003;348:651-6.

10. Gastmeier P. European perspective on surveillance. J Hosp Infect 2007;65(Suppl 2):159-64.

11. European Centre for Disease Prevention and Control (ECDC). Annual Epidemiological Report on Communicable Diseases in Europe 2008. Stockholm, March 2008. http://ecdc.europa.eu/en/ publications/publications/0812_sur_annual_epidemiological report 2008.pdf

12. Glasner $\mathrm{C}$, Albiger B, Buist $\mathrm{G}$, et al. The European Survey on Carbapenemase-Producing Enterobacteriaceae (EuSCAPE) working group. Carbapenemase-producing Enterobacteriaceae in Europe: a survey among national experts from 39 countries, February 2013 Euro Surveill 2013;18(28):pii=20525 doi:10.2807/1560-7917. ES2013.18.28.20525 